\title{
Self-Limited Cytomegalovirus Infection During Natalizumab Treatment for Multiple Sclerosis
}

\author{
Marina Rodrigues Lima ${ }^{1}$, Luís Arthur Brasil Gadelha Farias ${ }^{2}$, Maycon Fellipe da Ponte ${ }^{3}$, Luís Edmundo Teixeira de Arruda Furtado ${ }^{3}$ \\ ${ }^{1}$ Faculty of Medicine, University Center UNINTA, Sobral, Ceará, Brazil \\ ${ }^{2}$ Faculty of Medicine, Federal University of Ceará (UFC), Fortaleza, Ceará, Brazil \\ ${ }^{3}$ Internal Medicine Department, Hospital Regional Norte (HRN), Sobral, Ceará, Brazil
}

Received: 03/01/2019

Accepted: 09/01/2019

Published: 01/02/2019

How to cite this article: Lima M, Farias LA, da Ponte MF, Furdato LE. Self-limited cytomegalovirus infection during natalizumab treatment for multiple sclerosis. EJCRIM 2019;6: doi:10.12890/2019_001046.

Conflicts of Interests: The Authors declare that there are no competing interests.

Acknowledgements: We would like to thank the general practitioners from the Hospital Regional Norte, Fortaleza, Ceará, Brazil, for their help in collecting the clinical and therapeutic data from the patient.

Author Contributions: MRL and LABGF conceived and designed the study and wrote the paper. MFP collected the data and performed the analysis. MFP and LETAF performed the analysis and reviewed the manuscript.

This article is licensed under a Commons Attribution Non-Commercial 4.0 License

\section{ABSTRACT}

Natalizumab is indicated as monotherapy for the treatment of relapsing-remitting multiple sclerosis; it prevents outbreaks and delays the progression of physical disability. Here, we report the case of a 30-year-old patient with multiple sclerosis receiving natalizumab as monotherapy who subsequently developed self-limited cytomegalovirus disease. Cytomegalovirus infection has been reported during treatment with natalizumab, and in this study, we use new techniques to analyze the possible association of cytomegalovirus infection with natalizumab.

\section{LEARNING POINTS}

- Natalizumab is a humanized recombinant monoclonal antibody (IgG4k) against a4-integrin which promotes immunocompromise by blocking the adhesion interactions necessary for lymphocyte trafficking.

- Cytomegalovirus infection has been described during natalizumab treatment, although the pathogenesis and mechanisms are not complete understood.

- This case highlights the importance of awareness of this association and possible complications.

\section{KEYWORDS}

Infection, viruses, cytomegalovirus, natalizumab, multiple sclerosis

\section{INTRODUCTION}

Cytomegalovirus (CMV) is a ubiquitous human $\beta$-herpesvirus that is prevalent worldwide ${ }^{[1,2]}$. The outcome of CMV infection depends on the health of the infected individual. The majority of immunocompetent individuals infected with CMV are asymptomatic. However, clinical symptoms such as mononucleosis-like syndrome, which consists of an acute fever with marked lymphocytosis and lymphocytic atypia in $10 \%$ of cells, can be observed in some cases $^{[1,3,4]}$. 
Some patients, particularly those who are immunosuppressed, may develop infection with severe clinical symptoms. Treatment with natalizumab, which binds to a4-integrins, promotes immunocompromise by blocking the adhesion interactions necessary for lymphocyte trafficking, there by disrupting normal immunovigilance. Although numerous studies have discussed infections associated with natalizumab in the central nervous system (CNS), fewer studies have addressed infectious sequelae in other anatomical sites ${ }^{[5]}$.

Here, we report the case of a 30-year-old male patient with multiple sclerosis (MS) that evolved into CMV infection after treatment with natalizumab, focusing mainly on the clinical aspects, drug mechanisms, and side effects described in the literature.

\section{CASE DESCRIPTION}

A 30-year-old male patient from north-eastern Brazil, diagnosed with MS in 2010, who had a previous history of interferon use and had been treated with natalizumab (300 mg per month) for the previous 2 years (2016-2018), presented with sudden onset of pulsatile holocranial headache and photophobia associated with persistent myalgia and fever, with temperatures ranging from $38.3^{\circ} \mathrm{C}$ to $38.5^{\circ} \mathrm{C}$. In addition, he had difficulty walking, paraesthesia, and an isolated episode of diplopia. He denied urinary complaints, respiratory discomfort, angina, diarrhea or vomiting. The physical examination showed positive Romberg and Babinski signs, with no other significant observations. Red and white cell counts were within the normal range. $\mathrm{LDH}(1,452 \mathrm{IU} / \mathrm{I})$ and ferritin $(1,500 \mathrm{ng} / \mathrm{ml})$ levels were elevated.

Cerebral computer tomography (CT) did not reveal new lesions suggestive of disease activity (Fig.1). Anti-HIV 1 and 2, hepatitis B and C, venereal disease research laboratory (VDRL) and Toxoplasma serologies were negative. Tests for John Cunningham virus (JCV) were also negative. Blood culture was negative. Abdominal ultrasonography revealed homogeneous splenomegaly. An echocardiogram and endoscopy were normal. Other serological tests for mononucleosis-like diseases were requested and CMV immunoglobulin M and G (IgM and IgG) tested positive. The patient was diagnosed with cytomegalovirus disease associated with the use of natalizumab. No severe infections were found and treatment was administered on a symptomatic basis. Upon follow-up, we have determined that the patient has had a complete remission of symptoms.
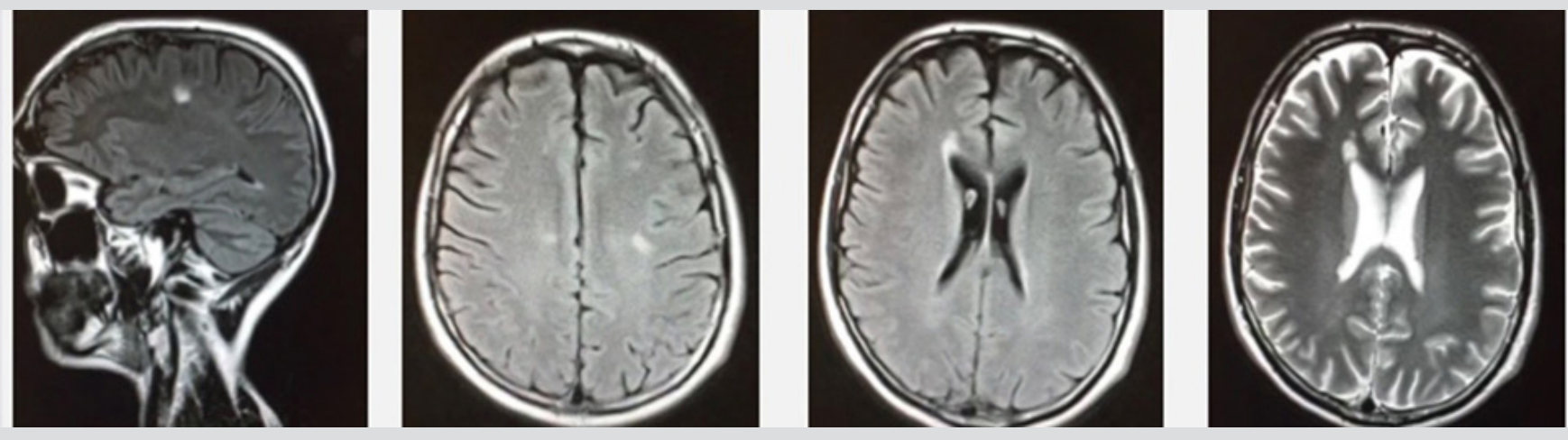

Figure 1. Cranial MRI revealing signal changes in the deep white matter at the septal callus interface and bridge to the left, suggestive of demyelinating disease

\section{DISCUSSION and CONCLUSION}

Natalizumab is a humanized recombinant monoclonal antibody (IgG4k) against a4-integrin, and is produced in a mouse cell line through recombinant DNA technology. It is usually prescribed as single-drug therapy for the treatment of relapsing-remitting MS to prevent outbreaks and delay the progression of physical disability ${ }^{[6]}$. Natalizumab has primarily been associated with progressive multifocal leukoencephalopathy secondary to JCV reactivation ${ }^{[7]}$. Consequently, clinical and laboratory assessments of a patient's immune status, including total leukocyte and T-lymphocyte counts, history of opportunistic infection and immunosuppressive therapy, and periodic JCV serological screening is recommended for patients taking this medication ${ }^{[8,9]}$. Other atypical infections, including herpes simplex, varicella zoster virus encephalitis/meningitis, as well as CMV infection, have also been described ${ }^{[10]}$.

In addition to the presented case, approximately 112 cases of CMV infection after the use of natalizumab have been described in the literature. Most of the reported patients were immunocompromised; however, several cases in immunocompetent patients have also been described $^{[11]}$. Other opportunistic infections of concern were found in patients with MS treated with anti-MS drugs such as alemtuzumab, ocrelizumab, fingolimod and mitoxantrone. 
Individual cases of Kaposi sarcoma, cutaneous histoplasmosis and CNS toxoplasmosis have been reported in non-HIV-infected patients with $\mathrm{MS}$ receiving fingolimod ${ }^{[12]}$. CMV was also identified in patients during MS treatment with alemtuzumab, suggesting a similar disease pathogenesis $^{[13]}$.

CMV infection is typically self-limiting and can be successfully treated in healthy patients; however, antiviral drugs have only been administered to patients who are immunosuppressed or have severe or persistent disease ${ }^{[14]}$. We hypothesize that CMV infection causes a mononucleosis-like disease in patients treated with natalizumab, by similarly disrupting normal immunosurveillance through lymphocyte a4-integrin blockade. However, further studies are needed to confirm the effect of natalizumab on CMV infection susceptibility.

The case discussed is relevant because it is a rare association of an opportunistic infection related to the use of natalizumab. In addition, it encourages further research to clarify this fact and validate the possible side effects related to this drug that have not been documented.

\section{REFERENCES}

1. Taylor GH. Cytomegalovirus. Am Fam Phys 2003;67:519-524.

2. Crough T, Khanna R. Immunobiology of human cytomegalovirus: from bench to bedside. Clin Microbiol Rev 2009;22:76-98.

3. Kano Y, Shiohara T. Current understanding of cytomegalovirus infection in immunocompetent individuals. J Dermatol Sci 2000;22:196-204.

4. Rafailidis PI, Mourtzoukou EG, Varbobitis IC, Falagas ME. Severe cytomegalovirus infection in apparently immunocompetent patients: a systematic review. Virol J 2008;5:47.

5. Gandhi MK, Khanna R. Human cytomegalovirus: clinical aspects, immuneregulation, and emerging treatments. Lancet Infect Dis 2004;4:725-738.

6. Polman CH, O'Connor PW, Havrdova E, et al. A randomized, placebo-controlled trial of natalizumab for relapsing multiple sclerosis. N Engl J Med 2006;354:899-910.

7. Warnke C, Olsson T, Hartung HP. PML: the dark side of immunotherapy in multiple sclerosis. Trends Pharmacol Sci 2015;36:799-801.

8. Gold R, Jawad A, Miller DH, et al. Expert opinion: guidelines for the use of natalizumab in multiple sclerosis patients previously treated with immunomodulating therapies. $J$ Neuroimmunol 2007;187:156-158.

9. Fernandez O. Best practice in the use of natalizumab in multiple sclerosis. Ther Adv Neurol Disord 2013;6:69-79.

10. Fine AJ, Sorbello A, Kortepeter C, Scarazzini L. Central nervous system herpes simplex and varicella zoster virus infections in natalizumab-treated patients. Clin Infect Dis 2013;57:849-852.

11. Crespo P, Dias N, Marques N, Saraiva da Cunha J. Gastritis as a manifestation of primary CMV infection in an immunocompetent host. BMJ Case Rep $2015 ; 2015$. pii:bcr2014206991.

12. Epstein DJ, Dunn J, Deresinski S. Infectious complications of multiple sclerosis therapies: implications for screening, prophylaxis, and management. Open Forum Infect Dis 2018;5(8):ofy174.

13. Clerico M, De Mercanti S, Artusi CA, Durelli L, Naismith R. Active CMV infection in two patients with multiple sclerosis treated with alemtuzumab. Multiple Sclerosis J 2017;23:874-876.

14. Goodgame RW. Gastrointestinal cytomegalovirus disease. Ann Intern Med 1993;119:924-935. 